\title{
Transmission Electron Microscopy of Catalytic Nanomaterials at Atomic Resolution
}

Sheng Dai ${ }^{1}$, Xingxu Yan ${ }^{1}$,Wenpei Gao ${ }^{1}$, Peter Tieu ${ }^{2}$, George W. Graham ${ }^{1,3}$ and Xiaoqing Pan ${ }^{1,4}$

1. Department of Materials Science and Engineering, University of California - Irvine, Irvine, CA, USA.

2. Department of Chemistry, University of California - Irvine, Irvine, CA, USA.

3. Department of Materials Science and Engineering, University of Michigan, Ann Arbor, MI, USA.

4. Department of Physics and Astronomy, University of California - Irvine, Irvine, CA, USA.

Atomic-scale insights into catalytic nanomaterials are critical for exploring the catalytic mechanism and the structure-property relationships. Detailed site-specific information about the structure and stability of nanocatalysts at atomic resolution, obtained by advanced transmission electron microscopy is an essential complement to other characterization techniques (e.g., x-ray photoelectron spectroscopy, x-ray absorption fine structure spectroscopy, et al.), which offer averaged information over larger length scales. In this talk, we present our TEM studies of various catalyst systems to highlight the advantages of TEM on catalysis research: (1) experimental and theoretical understanding of the anchoring site of single atom catalysts; (2) dynamic phase transformation from Pt precursor to Pt nanoclusters by low dose imaging; (3) observation of the growth of Pt-Ni nanoparticle under real gaseous environment.

Distinguishing single metal atoms in Z-contrast STEM images is crucial to identifying the location of catalytically active single atoms and their chemical bonding with oxide supports in single atom catalysts (SAC). However, this process becomes challenging when the atomic number difference between active atom and metal atom in the oxide support is small. The quantitative verification of single metal atoms depends on local thickness of the oxide support. Combining STEM image simulation with local thickness measurement, we are able to quantitatively identify active single metal atoms on various oxide supports. For the $\mathrm{Ir} / \mathrm{Fe}_{2} \mathrm{O}_{3}$ system $\left(Z_{\mathrm{Ir}}=77, Z_{\mathrm{Fe}}=26\right)$, the single Ir atom can be recognized when the thickness of $\mathrm{Fe}_{2} \mathrm{O}_{3}$ is less than $7 \mathrm{~nm}$, indicating that single $\mathrm{Ir}$ atoms can be easily observed at the edge of the support [1]. However, for $\mathrm{Ir} / \mathrm{WO}_{3}\left(Z_{\mathrm{II}}=77, Z_{\mathrm{W}}=74\right)$, the threshold thickness of $\mathrm{WO}_{3}$ is dramatically reduced to $1 \mathrm{~nm}$. In practice, we first performed low-loss EELS and convergent beam electron diffraction pattern to seek a region with thickness less than $1 \mathrm{~nm}$, and then acquired the STEM image as shown in Fig. 1a. The Ir atom exhibits $40 \%$ higher intensity than surrounding atomic columns in Fig. 1d, which is consistent with simulation results (Fig. 1c). The anchoring structure of $\mathrm{Ir} / \mathrm{WO}_{3}$ is shown in Fig. $1 b$, based on the STEM imaging analysis [2].

In addition, atomic-scale information about the kinetic growth of catalytic nanomaterials is critical for insights into growth mechanism and optimization of the synthesis process. Using state-of-the-art advanced electron microscopy and a direct electron detector, we achieved atomic resolution imaging of a Pt precursor compound and revealed the dynamics of its transformation into Pt nanoclusters [3]. Specifically, under an ultra-low electron dose in a double aberration corrected transmission electron microscope, the atomic structure of pristine $\mathrm{K}_{2} \mathrm{PtCl}_{4}$ crystallites was resolved by the CMOS-based direct electron detector. Then, the electron beam-induced dynamics of staged reduction of the Pt compound and subsequent nucleation of Pt clusters was revealed. Results show that the ionic bond between $\mathrm{K}^{+}$and $\mathrm{PtCl}_{4}{ }^{2-}$ is first broken, which is followed by $\left[\mathrm{PtCl}_{4}\right]^{2-}$ forming $\mathrm{PtCl}_{2}$; reduction of the dual-valent $\mathrm{Pt}$ then leads to the formation of Pt clusters. The entire solid state transition was thus deciphered in real time and real space. 
Furthermore, we utilized in situ atmospheric TEM to monitor the growth of octahedral $\mathrm{Pt}_{3} \mathrm{Ni}$ nanocatalysts under high purity $\mathrm{H}_{2} / \mathrm{CO}$ gas $\left(1.0\right.$ bar, $\left.\mathrm{H}_{2}: 4 \%, \mathrm{CO}: 96 \%\right)$, in which $\mathrm{H}_{2}$ acts as a reductant and $\mathrm{CO}$ acts as a capping agent for morphology control [4]. The sphere-like nucleus appeared from the reduction of the precursor containing $70 \mathrm{wt} \%$ of $\mathrm{Pt}(\mathrm{acac})_{2}$ and $30 \mathrm{wt} \%$ of $\mathrm{Ni}(\mathrm{acac})_{2}$ during the early growing stage. Then, facet formation and distinct lattice fringes were observed to evolve when the particle size slightly exceeded $1 \mathrm{~nm}$. Due to the distinct d-spacing, $\{001\}$ and $\{111\}$ facets were differentiated in the atomic-scale STEM images (Fig. 2), and the $\{111\}$ facets exhibited a significantly slower growth rate compared with the $\{001\}$ under the reaction condition, which was evidenced by a smaller number of atomic layers along the $<111>$ direction than along the $<001>$ one [5].

References:

[1] Y Zhao et al., P. Natl. Acad. Sci. USA 115 (2018), p. 2902.

[2] Y Zhao et al., ACS Central Sci. 4 (2018), p. 1166.

[3] W Gao et al., Sci. Adv. 5 (2019), p. eaau9590.

[4] X Shen et al. Nat. Commun. 9 (2018), p. 4485.

[5] The authors acknowledge the support of the University of California Irvine Materials Research Institute for the use of TEM facilities.

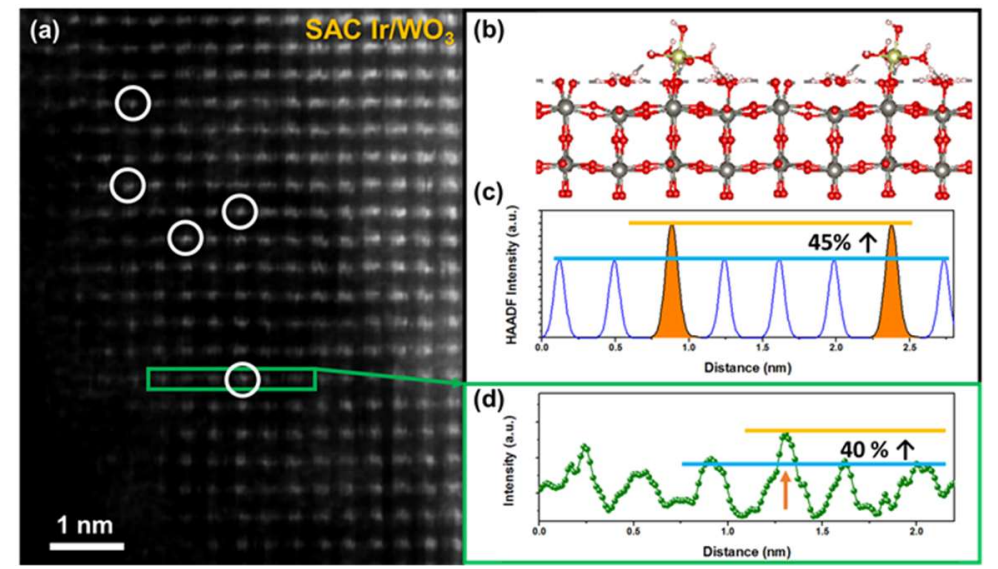

Figure 1. Identification of $\mathrm{Ir}$ atoms on $\mathrm{WO}_{3}$. (a) HAADF image along [020] axis. The white circles indicate single Ir atoms. Scale bar: $1 \mathrm{~nm}$. (b) Side view of atomic structure of $\mathrm{Ir}$ atoms on $(020) \mathrm{WO}_{3}$. Green ball for Ir, brown for $\mathrm{C}$, red for $\mathrm{O}$, white for $\mathrm{H}$, and gray for W. (c) Line scan profile of simulated HAADF intensity of Ir against the $\mathrm{WO}_{3}$ support with the same size as (b). The orange-shaded peaks indicate the position of Ir atoms in $\mathrm{Ir} / \mathrm{WO}_{3} \mathrm{SACs}$. (d) Line scan profile of the region highlighted in (a).

(a)

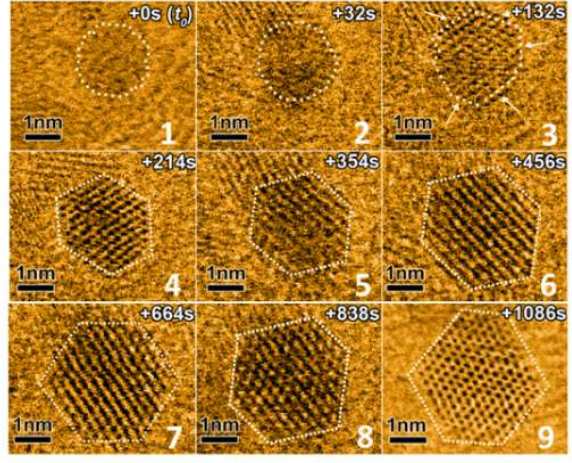

(b)

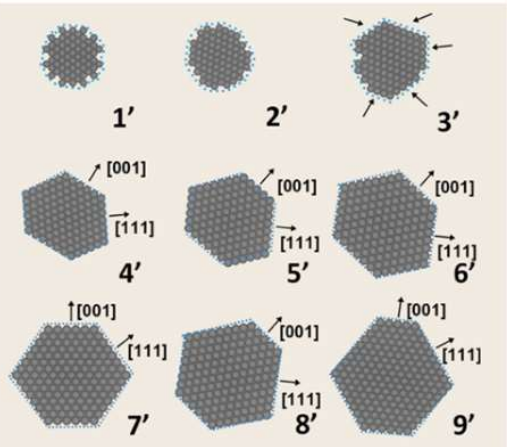

Figure 2. In situ observation of dynamic growth of $\mathrm{Pt}_{3} \mathrm{Ni}$ nanoparticles. (a) Sequential STEM images showing the growth of one $\mathrm{Pt}_{3} \mathrm{Ni}$ nanoparticle. (b) Corresponding 2D projection models of (a). 